\title{
Zum Umgang mit Zukunft in Organisationen - eine praxistheoretische Perspektive
}

\author{
Jochen Koch • Hannes Krämer • Andreas Reckwitz • Matthias Wenzel
}

Online publiziert: 22. September 2016

(C) Springer Fachmedien Wiesbaden 2016

Zusammenfassung Die Einsicht, dass Zukunft aufgrund ihrer Kontingenz unwissbar sei, hat Planung als zentrale Form der Zukunftsbearbeitung nachhaltig in Frage gestellt. Dennoch hat diese Erkenntnis und die daran anschließende Suche nach funktionalen Äquivalenten zu planerischen Steuerungshandlungen nicht dazu geführt, den Umgang mit Zukunft jenseits eines planungsbasierten Verständnisses zu denken und zu untersuchen. Zur Analyse organisationaler Zukunftsbearbeitung schlägt der vorliegende Beitrag deshalb eine praxistheoretische Perspektive vor. Dazu werden die analytischen Herausforderungen eines auf Zukunft ausgerichteten Forschungsprogramms herausgearbeitet und der praxistheoretische Ansatz vorgestellt. Eine solche Perspektive ermöglicht den Blick auf die vielfältigen, relational verschränkten und situativen Handlungsvollzüge zu richten, die das Kommende performativ hervorbringen und verarbeiten. Sie leistet damit einen wesentlichen Beitrag dazu, den Umgang mit Zukunft aus dem Zuschnitt eines genuinen Planungsproblems herauszuführen und neue Impulse zur Weiterentwicklung steuerungstheoretischer Ansätze zu liefern.

Schlüsselwörter Managementprozess $\cdot$ Planung $\cdot$ Praxistheorie $\cdot$ Zeit $\cdot$ Zukunft/ Zukünfte

\footnotetext{
J. Koch $(\triangle) \cdot$ M. Wenzel $(\triangle)$

Unternehmensführung und Organisation, Europa-Universität Viadrina, Große Scharrnstraße 59, 15230 Frankfurt (Oder), Deutschland

E-Mail:koch@europa-uni.de; mwenzel@europa-uni.de

H. Krämer $(\bowtie) \cdot$ A. Reckwitz $(\bowtie)$

Vergleichende Kultursoziologie, Europa-Universität Viadrina, Große Scharrnstraße 59, 15230 Frankfurt (Oder), Deutschland

E-Mail: kraemer@europa-uni.de; reckwitz@europa-uni.de
} 


\title{
On handling the future in organizations - a practice perspective
}

\begin{abstract}
The insight that the future is unknowable due to its contingency has destabilized planning actions as an allegedly safe form of handling the future. Nevertheless, this insight and the following search for functional equivalents to planning actions in corporate management has not led to rethinking and examining how organizations handle the future beyond a planning-based understanding. The present paper proposes a practice-theoretical perspective for the analysis of how organizations handle the future. For this purpose, the paper outlines the analytic challenges of a future-based research agenda and brings the practice-based approach into position. A practice perspective especially holds promising potentials because of its attention to the multifaceted, relationally bundled, and situated praxis through which the future is performatively generated and processed. By that, it provides an essential contribution to extending our understanding of handling the future as being more than a planning problem and sets new impulses for further developing theories of corporate management.
\end{abstract}

Keywords Management process $\cdot$ Planning $\cdot$ Practice theory $\cdot$ Time $\cdot$ Future/ futures

\section{Einleitung}

Organisationen - selbst als temporäre Einrichtung - sind immer zukunftsorientiert. Der Umgang mit dem Kommenden beschäftigt Organisationen jeglicher Art in sämtlichen Bereichen des wirtschaftlichen Lebens - von innovativen Start-ups bis Großkonzernen, und von Herstellungsbetrieben bis Dienstleistungsanbietern - und entscheidet maßgeblich über den Fortbestand und Untergang von Organisationen (Noss 2013). Beckerts (2016, S. 58) damit einhergehende Erkenntnis, dass ,the future matters just as much as history matters“, ist dabei keineswegs trivial, ist es doch jene Hervorbringung und Verarbeitung von Zukunft, die gegenwärtiges Handeln antreibt und damit organisationale, marktbezogene und gesellschaftliche Entwicklungen überhaupt erst auslöst. Ein besseres Verständnis derartiger Entwicklungen so die These - erfordert eine tiefergehende Analyse der Formen der Zukunftsbearbeitung, durch die Organisationen das Kommende hervorbringen und verarbeiten.

In der Managementforschung besteht zweifelsohne kein Mangel an zukunftsaffinen Diskursen. Im Gegenteil, Zukunft erscheint vielmehr omnipräsent und ubiquitär verhandelt unter Stichworten wie Möglichkeiten (Alvarez und Barney 2007), Risiko (Hardy und Maguire 2016), Unsicherheit (Weick und Sutcliffe 2007) und Optionen (Nadarajah et al. 2015). Dabei firmiert Zukunft insgesamt als ein prototypisches Synonym zugespitzter Kontingenzerfahrung, dem die Managementtheorie durch die Zentrierung planerischer Handlungen (vgl. Augier und Teece 2008; Hodgkinson und Healey 2011; Ordóñez et al. 2009) und der kontinuierlichen Weiterentwicklung und Verfeinerung von Planungstechniken (vgl. Hodgkinson und Wright 2002; Song et al. 2016) zu begegnen versucht. 
Mit der sich endgültig in der Spätmoderne manifestierenden Einsicht, dass Zukunft prinzipiell unwissbar ist, geht nun zwar auch die steuerungstheoretische Erkenntnis einher, dass es gerade nicht rational ist, der prinzipiellen Kontingenz des Zukünftigen mit immer noch rationaleren Planungstechnologien zu begegnen (vgl. Barry und Elmes 1997; Beckert 1996, 2013; March 1995; Mintzberg 1994). Zugleich bleibt jedoch der grundsätzliche Problembestand, den Umgang mit Zukunft als ein Planungsproblem, d. h. als die gegenwärtige Festlegung zukünftigen Geschehens zu begreifen, davon im Grunde unberührt. Die angestoßene Suche und Erforschung nach funktionalen Äquivalenten planerischer und - breiter gedacht - organisationaler (d.h. auch impliziter) Selektionsleistungen wird zwar nun im Konzert mit Kompensation und Systementwicklung gedacht (vgl. Schreyögg 1991). Die Bearbeitung von Zukunft selbst bleibt jedoch im Kern dabei eine selektionsorientierte, auch wenn die Selektionsstrukturen nun nicht mehr ausschließlich als ein Ergebnis intentionaler Planung gedacht werden. Wie Zukunft in Organisationen vorgestellt, bearbeitet, verarbeitet und mithin konstruiert wird, wird auf diese Weise dann eben auch nur selektiv, sprich als Selektionsstruktur sichtbar.

Freilich waren die theoretischen Möglichkeiten, die Engführung auf selektionsorientierte Formen der Zukunftsbearbeitung überwinden zu können, bisher auch noch kaum hinreichend entwickelt worden. Mit der Weiterentwicklung der Praxistheorie (vgl. u. a. Feldman und Orlikowski 2011; Nicolini 2013; Reckwitz 2002; Seidl und Whittington 2014; Vaara und Whittington 2012) hat sich diese Situation in den letzten Jahren jedoch - so unsere These - nachhaltig geändert. Durch die Fokussierung auf die konkreten Handlungsvollzüge nehmen praxistheoretische Perspektiven die vielzähligen und relational gebündelten Praktiken sowie deren Performanz in den Blick. Damit erscheint die Praxistheorie als eine sehr aussichtsreiche Kandidatin, die Zukunftsbearbeitung von und in Organisationen neu - und d.h. auch jenseits eines Selektionsproblems - zu denken, zu erforschen und zu analysieren.

Im folgenden Beitrag wird deshalb eine praxistheoretische Perspektive der $\mathrm{Zu}$ kunftsbearbeitung vorgeschlagen, welche die heterogene und komplexe Praxis der Bearbeitung, Markierung und Vergegenwärtigung von Zukunft in Organisationen in den Blick nimmt. Im Fokus steht somit die theoretische Beschreibung von ,Zukunftspraktiken“, d. h. jener Formen und Muster, mithilfe derer soziale Akteure ihre Zukunft imaginieren und Zukunftsvorstellungen im täglichen Handlungsvollzug verarbeiten. Indem sozialtheoretisch sowie methodologisch einem praxistheoretischen Theorieangebot gefolgt wird, rücken die Tätigkeiten, materialen Träger, (impliziten) Wissensformen und verbundenen Diskurse über Zukunft in Organisationen in den Fokus. Das Ziel dieses Beitrages ist es, diese Forschungsperspektive vorzustellen und ihre Attraktivität hinsichtlich der Analyse der Zukunftsbearbeitung zu verdeutlichen.

Dazu ist der Beitrag wie folgt aufgebaut: Zunächst werden die soziohistorischen Veränderungen in der Konzeption von Zukunft rekonstruiert. Dieser Wandel wird paradigmatisch deutlich in der Entwicklung moderner Organisationen und in der parallel dazu aufkommenden Managementtheorie, in welcher die prinzipielle Unwissbarkeit von Zukunft zunehmend in den Vordergrund getreten ist. Folglich zeigen diese Entwicklungen eine Reihe von Implikationen für die Analyse der Bearbeitung von Zukunft in Organisationen auf. Es wird deutlich, dass es theoretisch-konzep- 
tioneller Anstrengungen bedarf, um der Komplexität der Zukunftsbearbeitung und mithin des Zukunftsmanagements in Organisationen, die sich über die Grenzen eines Selektionsproblems erstreckt, gerecht zu werden. Der anschließende Abschnitt nimmt diese Ausgangslage auf und skizziert die praxistheoretische Perspektive als eine vielversprechende Möglichkeit, diese Komplexität einzufangen und theoretisch zu fassen. Nach der Einführung in grundlegende Begriffe und Konzepte einer Theorie sozialer Praktiken, wird der spezifische Blick auf Zeit und Zukunft erläutert. Traditionell präferierte die sozialwissenschaftliche Forschung zur Zeitlichkeit, und damit auch zur Zukunft, entweder eine subjektivistische oder objektivistische Erklärung. Die Praxistheorie integriert nun beide Perspektiven, indem sowohl nach der situativen Performanz der Zukunftshervorbringung als auch nach der strukturellen Prägung innerhalb derartiger Situationen gefragt wird. Zukunft wird somit keine rein innerlich-subjektive Erfahrungskategorie, wiewohl auch keine strukturdeterminierte Zwangsorientierung, sondern ein Phänomen der Praxis. Dabei sind drei Ebenen von Zeitlichkeit zu unterscheiden: (1) prinzipielle Zeitlichkeit jeglicher sozialer Praxis, (2) die Zeitlichkeit von Praktiken und (3) Zeitpraktiken im engeren Sinne. Während erstere die Gesamtheit von Praktiken umfasst, welche als Wiederholung von Ereignissen immer schon auf eine Zukunft und Vergangenheit verweisen, ist der zweite Zusammenhang von Praxis und Zeitlichkeit auf die hochspezifische temporale Strukturierung jeder einzelnen sozialen Praktik bezogen. Davon zu unterscheiden sind spezifische Zeitpraktiken, die im engeren Sinne darauf fokussiert und spezialisiert sind, die Zeit zu organisieren. Es ist besonders dieser letzte Typus, der für uns von besonderem Interesse ist. Wie dieser analytisch zu fassen ist, erläutert der nächste Abschnitt, indem die methodologischen Analyseprinzipien einer praxistheoretischen Untersuchung der Zukunftsbearbeitung benannt werden. Insbesondere werden hier die Performativität, Situationalität, Heterogenität und Relationalität von Zukunftspraktiken hervorgehoben. Diese Dimensionen bilden nicht nur das breitere praxistheoretische Forschungsprogramm paradigmatisch ab; sie liefern auch die Eckpfeiler praxistheoretischer Untersuchungen der Zukunftsbearbeitung, die der Managementforschung dazu verhelfen können, den Umgang mit dem Kommenden jenseits eines Selektionsproblems zu denken und zu analysieren. Den Abschluss des vorliegenden Beitrags bildet ein Ausblick auf derartige Zukunftsforschung und deren Chance, ausgehend von neueren Ansätzen der Unternehmenssteuerung weitere steuerungstheoretische Impulse in der Managementforschung zu setzen.

Der Beitrag verdeutlicht auch die Herausforderungen der in der Managementforschung weiterhin dominierenden Planungslogik im Hinblick auf die heutige Kontingenzerfahrung und zeigt, inwiefern eine praxistheoretische Perspektive den Umgang mit Zukunft in Organisationen analytisch zu durchdringen vermag. Eine solche Analyse stellt in der Forschung zu Zukunft in Organisationen ein Novum dar, da sie nicht in der alleinigen Analyse von Planungstechniken aufgeht. Vielmehr greift sie die vielseitigen zeit- und zukunftsbezogenen Aspekte gegenwärtiger Organisationswirklichkeiten auf und verdeutlicht das multiperspektivische Gefüge der Zukunftspraxis. In diesem Sinne und über den Beitrag weiter hinaus gedacht, stellt die hier in Anschlag gebrachte praxistheoretische Forschung ein transdisziplinäres Vernetzungsvokabular bereit, welches sich zunehmend fachübergreifend als sehr forschungs- und erkenntnisproduktiv erweist. 


\section{Rückblick: Die Vergangenheit der Zukunftsbearbeitung}

\subsection{Die Entwicklung des Umgangs mit Zukunft}

Zukunft ist zentral für das Leben in modernen Gesellschaften. Der Zeitmodus des Kommenden dient als Orientierungspunkt für gegenwärtiges Handeln - sei es als hoffnungsvolle Erwartung, strategisch-rationale Positionierung oder quasi-endzeitliche Befürchtung. Da sich das Zukünftige allerdings - anders als das Vergangene nicht auf eine vorgängige materielle Präsenz (z. B. Dokumente, Archive) verlassen kann, muss das Kommende in einem besonderen Maße hervorgebracht, das heißt mit Hilfe von Praktiken und Repräsentationen adressiert, imaginiert und bearbeitet werden. Derartiges Vergegenwärtigen der Zukunft ist kein exklusives Problem kommender Zeit(en), sondern bezieht seine Aktualität aus der Präsenz und Praxis des Zukünftigen in der Gegenwart. Dies erhält vor dem Hintergrund der Gegenwartsgesellschaft eine herausgehobene Bedeutung, da sich in der Spätmoderne Zukunft pluralisiert und damit verschiedene, auch konkurrierende, sich überlagernde und verstärkende Konzeptionen von Zukunft deutlich werden. Mit anderen Worten: Zukunft wird in der Gegenwartsgesellschaft verstärkt zu einem Problem.

Dies ist in der „Geschichte der Zukunft“ keine Selbstverständlichkeit. Erst mit dem Übergang zur Epoche der Moderne ab der zweiten Hälfte des 18. Jahrhunderts wird das Konzept „Zukunft“ als ein kontingenter Zeitmodus konzipiert, der nun nicht mehr einer rein religiösen Chronoteleologie folgt und sich an metaphysischen Endlichkeitsvorstellungen orientiert (vgl. Koselleck 1989), sondern als Ergebnis zahlreicher sozialer, wissenschaftlicher und technischer Einflüsse betrachtet wird. Zukunft gerät in der Moderne als etwas Veränderliches in den Blick, was jenseits der Wiederkehr des Immergleichen (wie etwa der Zyklus der Jahreszeiten) nicht nur als wandelbare, sondern ebenso als beeinflussbare Kategorie erscheint (vgl. Hölscher 1999; Minois 1998). Diese Entwicklung lässt sich innerhalb der Moderne noch weiter ausdifferenzieren. Für den Wandel von Organisationen und ihrer Steuerung sind die einflussreichen Veränderungen im Übergang von einer klassischen Moderne zur Spätmoderne von besonderem Interesse (vgl. Nowotny 1989; Rosa 2005). Diese klassische Moderne (ca. 1890-1970), Beck (1986) spricht hier von einer „,ersten Moderne“ und Wagner (1994) von der ,organisierten Moderne“, weist - pauschal gesagt - eine lineare Zeitkonzeption auf, welche als stringente (Fort-) Entwicklung von der Vergangenheit in die Zukunft begriffen werden kann. Zukunft gilt zwar als etwas Neues, allerdings nicht als dramatischer Strukturbruch mit der Gegenwart, sondern vielmehr als eine plan- und beherrschbare Zeitdimension. Mithilfe voranschreitender Kalkulation, Rationalisierung, Professionalisierung und entsprechender Verfahren wie etwa der Prognostik, Szenariotechnik, Trendanalyse und weiterer Planungsmethoden wird die Unsicherheit der Zukunft bearbeitbar und letztlich ent-problematisiert.

Ein derartiger Gestaltungsoptimismus durchzieht auch die Vorstellungen von Organisationen und Management. Organisationen der klassischen Moderne folgen zwar idealtypisch, aber gleichwohl wirkmächtig - Webers (1922) Bürokratiemodell formaler Rationalisierung und Standardisierung. Als derartig rationale Systeme eröffnen Organisationen einen spezifischen Blick auf das Kommende (vgl. allgemein 
dazu Adam 1990, S. 104 ff.; Reckwitz 2016): Zukunft gerät als Ergebnis eines kontrollierbaren Prozesses in den Blick. Immer mehr organisationale Praktiken sind einer zeitlichen Kontrolle unterworfen, die sich in standardisierten Zeiteinheiten, vorgesehenen Bearbeitungsdauern, Sequenzen, Synchronisationen und gesteuerten Geschwindigkeiten niederschlägt. Dies gilt auch für die Zukunft, welche in Plänen und Zielerreichungen gefasst und somit auf eine für die Moderne typische Art und Weise komprimiert wird. Für Organisationen bedeutet eine solche Kompression der Zeit eine Temposteigerung von Ereignissen, beispielsweise eine Beschleunigung von Produktions- oder Vertriebsprozessen. Die Zukunft rückt aus der unscharfen Ferne in eine erkennbare Nähe. In diesem Kontext wird Zeit und auch Zukunft zu einer Ware. Nicht nur als knappes Gut in der Gegenwart, sondern auch als (beispielsweise technologischer) Vorsprung gegenüber anderen Akteuren, lassen sich ökonomische Zugriffe auf Zukunft verzeichnen. Über kommende Entwicklungen im Bilde zu sein, diese möglicherweise gar zu gestalten, erscheint als ein ökonomischer Vorteil, auf den hin die Organisation auszurichten ist - sei es über die systematische Nutzung von Vorhersagetechniken (wie etwa Prognosen) oder die Einführung von Forschungsabteilungen. Eine derartige Organisationform verweist auf eine Kolonialisierung der Zeit in die Zukunft hin. Zukunft ist abhängig von der Entscheidung in der Gegenwart und lässt sich im Rahmen eines formal-rationalistischen Modells planen. Das Kommende wird als eine leere Fläche imaginiert, auf die sich Ziele projizieren lassen und die sich - das ist entscheidend - mithilfe von Planungsverfahren in Angriff nehmen lassen.

Diese Zukunftssicherheit gerät allerdings in der spätmodernen Gegenwartsgesellschaft (ab ca. 1970) ins Wanken, auch wenn dies nicht als klarer Wechsel, sondern als eine Verschränkung konkurrierender und überlagernder Zeithorizonte vergegenwärtigt werden muss. Zeitanalytische Studien zeigen, dass sich die Bedeutung zeitlicher Ungewissheit verstärkt und Nichtwissen, mithin Risiko, immer präsenter werden (Böhle et al. 2004; Bröckling 2008; Strohschneider und von der Weth 2002). Zugleich pluralisieren sich verschiedene Zeit- und Zukunftskonzepte. So kann nicht mehr auf ein metazeitliches, prästabiles Zeitkonzept zurückgegriffen werden. Vielmehr lässt sich eine „Verzeitlichung der Zeit“ beobachten, nachdem in jeweiligen gesellschaftlichen Feldern und Situationen entschieden wird, welche Rhythmen, Durées, Reihenfolgen von Ereignissen und Handlungen wichtig werden (vgl. Rosa 2005). Diese Zeitpluralität wird erneut als Zukunftsunwissenheit deutlich, aber ohne die Gewissheit adäquater Instrumente der Zukunftsbearbeitung. Zukunft wird damit zu einer deutlichen Grenze der Jetztzeit, zu einem potentiell radikalen Strukturbruch mit der Gegenwart.

\subsection{Zur Rolle von Zukunft in der Managementforschung}

Entgegen dieser spätmodernen Erfahrung hat die Managementforschung in der Breite sehr lange an einem linearen Zeitkonzept festgehalten und führt die Vorstellung eines bearbeitbaren und strukturierbaren Planungshorizonts teilweise auch heute noch in unterschiedlichen Formen fort. Gerade die frühe Managementforschung nahm die Idee der Beherrschbarkeit von Zukunft in linearen Ansätzen der Zukunftsbearbeitung auf. So hat beispielsweise Chandler (1962) mit seiner bekannten These 
„structure follows strategy“ darauf hingewiesen, dass erfolgreiches zukünftiges Handeln zu planen sei. Auch fanden eine Reihe von Planungstechnologien in der frühen Managementforschung ihren Ursprung (vgl. z. B. Ansoff 1965), welche angesichts erkannter Herausforderungen der Zukunftsbearbeitung fortlaufend weiterentwickelt und verfeinert wurden (vgl. Hodgkinson und Wright 2002; Song et al. 2016). Dass diese Entwicklungen keineswegs zu einer Abkehr von der linearen Zeitkonzeption in der Managementforschung geführt haben, kommt u. a. in gegenwärtig verwendeten Konzepten wie Tempo (Pacheco-de-Almeida et al. 2015), Geschwindigkeit (Nadkarni et al. 2016), ,timing“ (Hawk und Yeung 2015) und Beschleunigung (vgl. Clarysse et al. 2014) zum Tragen. Die lineare Zeitkonzeption trifft insbesondere für alle an linear-rationalen Entscheidungsmodellen orientierten Ansätze und der damit verbundenen Steuerungslogik zu (vgl. Hodgkinson und Healey 2011). Beispielsweise beruht der neuere ,dynamic capabilities“-Ansatz (vgl. u. a. Teece 2007) weiterhin auf der ,anticipation of uncertain futures“ (Augier und Teece 2008, S. 1192) durch ,up-front planning“" (Arend 2015, S. 79). Auch tragen viele Controlling- und Zielsteuerungskonzepte bei genauerem Blick nach wie vor die Insignien eines ungestörten Planungsoptimismus in sich, letztlich doch durch gezielte Bearbeitung und verbesserte Instrumente Zukunftskontingenz in den Griff zu bekommen (vgl. Ordóñez et al. 2009). Solche linear-rationalen Modelle der Zukunftsbearbeitung erleben nicht zuletzt durch die zunehmende Verfügbarkeit und computergestützte Verarbeitung von Daten auch in informationstechnologieorientierten Bereichen wie der Wirtschaftsinformatik eine deutliche Renaissance (vgl. Guericke et al. 2012).

Dieser implizite Planungsoptimismus mag angesichts der unhintergehbaren Kontingenzerfahrung organisatorischen Zukunftshandelns überraschen, stellt aber gerade aus der Perspektive der Unternehmenspraxis ein durchaus verständliches Desiderat dar. Unter anderem hat die praxistheoretische Managementforschung (vgl. Abdallah und Langley 2014; Jarzabkowski und Balogun 2009; Jarzabkowski und Kaplan 2015) gezeigt, dass Pläne, d. h. Festlegung für die Zukunft, nach wie vor die Grundlage vieler organisationaler Prozesse konstituieren. Obwohl diese Form der Zukunftsbearbeitung in gewissen Belangen Ähnlichkeit zu Horoskopen und religiösen Predigten aufweist (vgl. Curnot et al. 2012), entsprechen Unternehmen damit einer weit verbreiteten Rationalitätsanforderung, die sowohl in Quartalsberichten als auch in anderen legitimationsorientierten Diskursen erwartet wird und sich als solche auch bewährt, d. h. zur Attribution von Legitimität führt (vgl. Vaara et al. 2010). Dies trifft selbst für Teile der Entrepreneurship- und Startup-Szene zu, in der auf der einen Seite die Idee des „effectuation“ propagiert wird (vgl. Sarasvathy 2001), welche prinzipiell gegen die Planungslogik gerichtet ist, auf der anderen Seite aber der sogenannte „Business Plan“ und darin enthaltene detaillierte Aussagen u. a. zu zukünftigen Zahlungsströmen weiterhin eine wichtige Größe darstellt (vgl. Beckert 2016; Brinckmann und Sung 2015). Die Idee jedenfalls, dass man sich aufgrund der Kontingenzerfahrung des Kommenden nicht in der Lage sieht, eine klare Aussage darüber zu treffen, wo man in Zukunft stehen wird, scheint nach wie vor trotz aller Fallibilität von Plänen, grundsätzlich nicht akzeptabel zu sein (Clegg et al. 2005), besteht doch die Aufgabe des Managements gerade darin, zu steuern und mithin Steuerbarkeit herzustellen (vgl. Nag et al. 2007). Insofern erfüllen Planungsverfahren das Versprechen einer Gestaltbarkeit der Zukunft und ermöglichen damit selbst 
bei Eintreten anderer Zukünfte eine wesentliche Funktion in der Gegenwart, die mit dem Begriff der Legitimationsfassade (vgl. z. B. Meyer und Rowan 1977) - da diese unausweichlich erscheint - im Grunde genommen eine zu negative Konnotation erhält. Es ist in diesem Zusammenhang deshalb mehr als eine Koinzidenz wissenschaftlicher Entwicklung, dass das Phänomen eines Auseinanderfallens von action und talk (vgl. Brunsson 1989) und die zunehmende Bedeutung von Legitimation und Legitimationsdiskursen für die Managementforschung (vgl. Joutsenvirta und Vaara 2015; Vaara et al. 2004; Vaara und Tienari 2008) einhergehen mit der oben geschilderten zunehmenden Einsicht einer grundlegenden Kontingenzerfahrung.

Jedoch findet diese Kontingenzerfahrung insbesondere in neueren reflexiven Ansätzen Beachtung, die die Steuerung von Unternehmen gerade auch im Hinblick auf ihre Nicht-Steuerbarkeit konzeptionalisieren, indem sie das eindeutige ,So wird es sein!“ durch das mehrdeutige „Es könnte so sein, es könnte aber auch anders sein!““ ersetzen (vgl. Kasper et al. 1999; Koch 2003; Ortmann 2003, 2013; Schreyögg 1991; Willke 2001). In diesen Ansätzen wird Zukunft vornehmlich als eine Frage der Grenze von Wissen, als grundlegendes, d. h. unhintergehbares Selektionsrisiko für kommende Entwicklungen konzipiert, das nicht selbst wiederum planerisch kompensiert werden kann, sondern nach funktional äquivalenten Steuerungsinstrumenten sucht (vgl. dazu auch bereits Luhmann 1973).

Einen der umfassendsten Ansätze für eine solche Steuerungsvorstellung hat Georg Schreyögg (vgl. Schreyögg 1991; Schreyögg und Steinmann 1987) mit seinem modernen Managementprozess vorgelegt und damit die hier geschilderte Kontingenzerfahrung in ein konzises theoretisches Konzept gegossen. Ausgangspunkt seiner Überlegungen ist der klassische Managementprozess und die Kritik an dessen immanenter, plandeterminierter Steuerungslogik, in welcher die gegenwärtige Festlegung zukünftigen Geschehens durch planerische Handlungen das Steuerungspotenzial aller weiteren Managementfunktionen (d.h. Organisation, Personaleinsatz, Führung und Kontrolle) unmittelbar und in einer linearen und zyklischen (Zeit-)Logik ableitet. Somit kommt in der klassischen Vorstellung des Managementprozesses auch alleine der Planung die Funktion der Zukunftsbearbeitung zu, d. h., nur in der Planung findet im Prinzip eine Bearbeitung von Zukunft statt, wohingegen allen weiteren Steuerungshandlungen kein eigenes Zukunftsbearbeitungspotenzial zugesprochen wird. Vor dem Hintergrund systemtheoretischer Überlegungen hat Schreyögg (1991) die mit dem klassischen Managementprozess einhergehende Steuerungslogik grundlegend revidiert und neu gefasst. Die zentrale Prämisse dieser Neukonzeption stellt die Einsicht in die grundlegende Komplexität der Umwelt und die unhintergehbare Kontingenz der Zukunft dar. In diesem Sinne ist die Festlegung einer Sollordnung immer nur als ein Entwurf zu denken und als solcher könnte diese immer auch anders aussehen, d.h., jegliche konkrete Form von Sollordnung ist immer selektiv. Insofern ist jede Sollordnung risikobehaftet und potenziell korrekturbedürftig. Aus Steuerungsperspektive sind daher Maßnahmen erforderlich, die das durch Kontingenz getriebene Risiko von Überraschungen kompensieren und nicht tragfähige Sollordnungen nach den aktuellen Steuerungserfordernissen und somit zeitinvariant verändern und weiterentwickeln. Damit werden Selektion, Kompensation und Systementwicklung als abstrakte Systemfunktionen zu zentralen Eckpfeilern des neuen Steuerungsdenkens, das - und darin liegt nun die wesentliche Idee - (a) nicht mehr 
allein durch die Planung und (b) nicht mehr in einer linearen und zyklischen (Zeit-) Logik gedacht wird.

Auf theoretischer Ebene wurden derartige Entwicklungen von der avancierten Managementforschung aufgegriffen (vgl. z. B. Boje 1995; Chia 1995). Neuere Ansätze konzeptionalisieren die Steuerung von Organisationen mit Begrifflichkeiten wie ,strategy without design“ (Chia und Holt 2009), ,emergent strategies“ (Mintzberg und Waters 1985; Mirabeau und Maguire 2014), ,,autonomous strategic actions“ (Burgelman 2002; Burgelman und Grove 2007), ,edge of chaos“ (Brown und Eisenhardt 1997) und sogar „organizations as wonderland“ (McCabe 2016). Dies geschieht gerade auch mit Blick auf ihre Nichtsteuerbarkeit, womit deutlich wird, dass das Kommende nicht länger aus einer vermeintlichen Sicherheit perfekter Planung und technischer Rationalität bestimmt werden kann. Eine derartige Skepsis gegenüber der Vorhersehbarkeit und Wissbarkeit des Kommenden resultiert organisatorisch in einer intensivierten Gegenwartsorientierung. In diesem Kontext der „instantaneous time“ (Urry 2000) erhält die Suche nach alternativen Verfahren der Zukunftsbearbeitung neue Bedeutung. Ebenso lässt sich eine Gleichzeitigkeit verschiedener Zukunftskonzepte verzeichnen. So finden innerhalb von Organisationen sowohl Planungsverfahren alten Musters als auch neuere kontingenzoffenere Verfahren Platz.

Vor dem Hintergrund neuerer Ansätze der Unternehmenssteuerung wird deutlich, dass auch die Zukunftsbearbeitung letztlich vollkommen neu und sehr viel komplexer $-\mathrm{d}$. h. sich nicht ausschließlich in planerischen und/oder emergenter Handlungen erschöpfend - gedacht werden muss. Denn unbeleuchtet bleiben die empirisch vielgestaltigen Arten und Weisen des Umgangs mit Zukunft jenseits einer prästabilen Konzeption von Entscheidungsprozessen. Auf der Ebene der Organisationswirklichkeit bedeutet dies, dass verschiedene, ja sogar konkurrierende Praktiken der Zukunftsbearbeitung deutlich werden können (vgl. Costas und Grey 2014; Kaplan und Orlikowski 2013; Schultz und Hernes 2013). Zugleich wird eine derartige Zukunft im Plural - Luhmann (1984) spricht damit prinzipiell von „Zukünften“ - auch an verschiedene Situationen rückgebunden und erhält somit eine performative Dimension. Gegenwärtige Zukunft, also die Präsenz des Zukünftigen in der jetzigen Handlungs- und Erlebnisunmittelbarkeit, ist immer auf ein performatives Element angewiesen. Sie wird erst hergestellt (vgl. Beckert 2013, 2016).

Für die Managementforschung zeigt diese Ausgangslage hinsichtlich der Untersuchung von Zukunftsbearbeitung drei analytische Konsequenzen. Erstens muss sich eine solche Perspektive der Situativität und damit auch der Performanz gegenwärtiger Zukunftskonstruktionen zuwenden (vgl. auch Kaplan und Orlikowski 2013). Sie muss danach fragen, wie konkret in der Gegenwart Zukunft bearbeitet wird und welche Zukünfte dabei entstehen. Es macht demnach einen Unterschied für die Zukunft selbst, mit welchen Mitteln sie imaginiert wird. Sind es beispielsweise prognostisch-probalistische Verfahren, werden existierende Trends weiterberechnet; bei visionären Zukunftserzählungen hingegen geht es gerade explizit um den Bruch mit gegenwärtigen und bekannten Zukunftsnarrativen. Somit lassen beide Verfahren differente Vorstellungen über das Kommende entstehen und sind auch nur noch schwerlich unter der Rubrik der Selektionsstruktur einheitlich zu subsumieren. 
Daher muss sich, zweitens, eine zukunftsinteressierte Managementforschung von den konkreten Figurationen der Zukunftsbearbeitung überraschen lassen. Das bedeutet, nicht prinzipiell von der Bearbeitung des Kommenden durch planerische Handlungen auszugehen, sondern immer auch die Kontingenz der Zukunft zu berücksichtigen. Dadurch wird das, was kommt, nicht nur zu einer Frage jetziger Selektionen, sondern auch anderer Einflüsse und Entwicklungen, die nicht vorhersehbar sind und kompensiert werden müssen. Dies führt zu einer Vielgestaltigkeit möglicher Formen der Zukunftsbearbeitung im praktischen Handlungsvollzug, die es zu untersuchen gilt.

Dies aufgreifend muss drittens eine Perspektive auf die Zukunftsbearbeitung die Relationalität, also das Verhältnis der zahlreichen Praktiken zueinander in den Blick nehmen, mit denen in Organisationen Aufschluss über das Kommende erlangt wird. Denn die Zukunftsformen in der Organisation setzen sich einerseits aus zahlreichen diskursiven und nicht-diskursiven Praktiken zusammen und sind andererseits rekursiv eingebunden in ein weites Netz verschiedener Einflüsse, welche so unterschiedliche Aspekte wie organisationsinterne und -externe Normen und habituelle Zwänge umfassen (vgl. auch Howard-Grenville 2005).

Einen vielversprechenden Ansatz, der über die theoretischen Mittel verfügt, einen solchen Blick auf komplexe Organisationswirklichkeiten zu ermöglichen, stellt unseres Erachtens die Theorie sozialer Praktiken dar. Den analytischen Fokus einer solchen Theorie sozialer Praktiken gilt es im Folgenden genauer zu umschreiben.

\section{In situ: Grundlagen eines praxistheoretischen Verständnisses der Zukunftsbearbeitung}

\subsection{Praxistheorie: Eine Einführung}

Unter der Bezeichnung ,Theorie sozialer Praktiken“ oder auch „Praxistheorie“ firmieren in den letzten Jahren theoretische Ansätze, die sich durch eine Art „Familienähnlichkeit" (Wittgenstein) auszeichnen (vgl. Reckwitz 2002; Feldman und Orlikowski 2011; Vaara und Whittington 2012; Nicolini 2013; Seidl und Whittington 2014). ${ }^{1}$ Das heißt, dass sich die Praxistheorie zwar aus unterschiedlichen Theorierichtungen speist - das umfasst Ansätze wie Bourdieus Praxeologie, die Ethnomethodologie, sozialkonstruktivistische Zugänge verschiedener Forschungsrichtungen wie auch die sozialphilosophischen Überlegungen von Theodore Schatzki oder Bruno Latour aber durchaus Ähnlichkeiten im theoretischen Zugriff vorliegen. Die Praxistheorie besticht damit nicht durch ein umfassendes Theorievokabular, sondern zeichnet sich eher durch einen schlanken Begriffsapparat aus, der für synchrone und diachrone empirische Forschungen in verschiedenen Feldern Anschluss bietet.

Der Ausgangspunkt der Praxistheorie besteht darin, als kleinste Einheit der Sozialtheorie etwas anzunehmen, was von vornherein strukturierte Aktivität und damit Handeln und Struktur zugleich ist: die soziale Praxis bzw. die sozialen Praktiken. Auf einer sozialtheoretischen Ebene setzt die Praxistheorie damit weder am in-

\footnotetext{
1 Zu einzelnen Aspekten dieses Abschnitts siehe auch Reckwitz (2016).
} 
tentionalen Handlungsakt noch am Determinismus sozialer Strukturen und Regeln an, sondern betont die routinierten Verhaltens- und Handlungsabläufe, welche eben durch eine gewisse Routine auf die Trajektorie ihrer Entstehung verweisen und diese Strukturen und Regeln im Handlungsvollzug erst (re-)produzieren, zugleich aber in Situationen eingebunden und damit auch veränderlich sind. Die vollzogenen Aktivitäten in eben solchen Situationen sind als Praktiken zu begreifen, wobei es gerade eine doppelte Form von Materialität ist, die diese Praktiken auszeichnet - nämlich umfassen Praktiken sowohl die menschlichen Körper als auch die Artefakte, welche beide für die Aufführung der sozialen Praxis maßgeblich nötig sind. Das heißt, dass Personen nicht das aktivistische Zentrum, zumindest nicht das einzige Zentrum einer sozialen Praxis sind. Soziale Praktiken bestehen eben aus dem Zusammenspiel von teilnehmenden „Partizipanden“ (Hirschauer 2004).

Die Sozialwelt, so eine weitere praxistheoretische Basisannahme, besteht aus einem komplexen Gefüge heterogener, miteinander verbundener Praktiken. Beispiele für solche Praktiken als Typen von Aktivitäten sind etwa Praktiken der Kooperation (in Organisationen), also die Arten und Weisen der gemeinsamen Bearbeitung einer Aufgabe. Als Unterformen derartiger Kooperationspraktiken können Meetings, Absprachen, Feedbackrunden etc. gelten. Dabei gibt es Praktiken, die in gewissen Bereichen immer wieder auftreten. So genannte dispersed practices (Schatzki 1996, S. 91 ff.) können etwa Praktiken des Meetings sein, welche sich eben in verschiedenen sozialen Feldern finden, etwa ebenso in Hochschulen wie in Unternehmen. Demgegenüber sind die so genannten integrated practices (Schatzki 1996, S. 98 ff.) enger an einzelne Felder gekoppelt; ein Beispiel wäre das Führen eines wissenschaftlichen Beweises.

Wiederum mit Theodore Schatzki gesprochen bilden Praktiken einen ,nexus of doings and sayings“ (Schatzki 2012, S. 15), was bedeutet, dass sie zum einen durch materiale Träger, eben Körper und Artefakte, auf- und ausgeführt werden. Zum anderen verweist dies auch auf eine Dimension von Wissen, da die Praktiken immer als gekonnte und in der Regel (wieder-)erkennbare Formen des Doings sind. Dieses Wissen, welches in der Regel als implizites Wissen auftritt, organisiert die jeweiligen Praktiken. Derartige Organisationen von Aktivitäten werden als praktische Ordnungen deutlich, die im Handlungsvollzug relational verschränkt sind.

Praktiken sind in diesem Verständnis immer kulturelle Praktiken, indem sie spezifische kulturelle Ordnungen des Wissens enthalten. Sie sind immer materiale Praktiken, indem sie in zwei Sorten von Materialität, menschlichen Körpern und Dingen, verankert sind. Und sie sind immer soziale Praktiken, insofern sie über räumliche und zeitliche Grenzen hinweg, das heißt an verschiedenen Orten zu unterschiedlichen Zeitpunkten, getragen von diversen Individuen immer wieder in ähnlicher Form hervorgebracht werden. Die praxistheoretische Perspektive setzt damit nicht an den handelnden Subjekten an, sie betreibt vielmehr ihre eigene Version einer Dezentrierung des Subjekts: Die sozialen Praktiken bedienen sich gewissermaßen der Subjekte ihres Körpers und ihres Geistes, die auf eine bestimmte Weise mit Foucault gesprochen ,subjektiviert“ werden. 


\subsection{Zur Form von Zukunftspraktiken: Zwischen Objektivismus und Subjektivismus}

Eine Praxistheorie der Zukunft operiert jenseits der konventionellen sozialwissenschaftlichen Deutungsangebote zum Thema Zeitlichkeit. Mit dem oben beschriebenen Diskurs zur (Nicht-)Linearität von Zukunftsvorstellungen eng verknüpft, wurde Zeit in den Sozial- und Wirtschaftswissenschaften implizit entweder als eine objektive oder eine subjektive Kategorie verstanden (vgl. Ancona et al. 2001; Bakken et al. 2013; Bluedorn 2002; Hydle 2015; Orlikowski und Yates 2002; vgl. dazu insgesamt auch Reckwitz 2016). Das objektivistische Verständnis fasst Zeit als einen neutralen Hintergrund, vor dem Ereignisse ablaufen. Zeit ist demnach als standardisierte, messbare und vergleichbare Einheit für alle Abläufe prinzipiell dieselbe. Ein solcher Temporalmodus verweist auf Quantität und wird im Kontext der Managementforschung zentral im Taylorismus aufgegriffen. Danach werden Arbeitsschritte in messbare Zeiteinheiten zergliedert und auf ihre Leistung hin mit anderen verglichen - so wird dann die Zeitdauer zum Öffnen von Schubladen gemessen etc. In Planungstheorien schlägt sich ein derartiges Zeitkonzept im Aufkommen verschiedener Pläne nieder, die allesamt in gleicher Art und Weise Zeit fassen. Demgegenüber findet sich ein subjektivistisches Zeitverständnis, welches den individuellen, sozialen und kulturellen Charakter von Zeitlichkeit in den Blick nimmt. Hierbei geht es eher um Zeit als Qualität, also um die jeweilig spezifischen Formen des Zeiterlebens und Zeitbegreifens. Zeit wird demnach vor allem als eine Leistung der Wahrnehmung begriffen, es geht beispielsweise darum, unterschiedliche Dauern zu erfassen. Das Öffnen einer Schublade ,dauert“ dann entsprechend länger, wenn beispielsweise der Tag schon weiter fortgeschritten ist. Eine solche subjektivistische Kategorie von Zeit ist aber keineswegs auf ein Individuum beschränkt. Ebenso sind soziale Gruppen denkbar, in denen Zeitabläufe anders wahrgenommen werden - etwa zeichnet sich die ,Zeitkultur“ in Sterneküchen (vgl. Wenzel et al. 2016) und Werbeagenturen (vgl. Krämer 2014) durch eine höhere Kurzfristigkeit, Spontaneität und Frequenzwechsel aus, als dies in Bestattungshäusern (vgl. Wenzel 2015) und Zeitungsverlagen (vgl. Koch 2011) mit ihrer Orientierung an temporaler Stabilität der Fall ist.

Eine praxistheoretische Perspektive nimmt nun eine Stellung zwischen und jenseits dieser objektiven Zeitstruktur und einer subjektivistischen Position ein. Zeit ist demnach keine objektive Rahmenbedingung, aber auch nicht lediglich ein subjektives Zeitbewusstsein, sondern Resultat und Bestandteil der jeweiligen Praktik: Praktiken wirken vielmehr verzeitlichend, das heißt, sie bringen ihre eigene temporale Struktur hervor.

Eine solche Perspektive bedeutet keineswegs eine Verortung auf Seiten eines Subjektivismus'. Entgegen der Vorstellung (kollektiver) mentaler Strukturen, die die Wahrnehmung von Zeitlichkeit strukturieren, betont die praxistheoretische Argumentation die Strukturierung von Zeit durch entsprechende Praktiken. Zeit ist demnach nicht der Praxis vorgängig, weil sie etwa in mentalen Dispositionen sedimentiert ist, sondern sie ist, aus einer praxistheoretischen Perspektive, das Ergebnis von ausgeführten Aktivitäten, die etwa einen bestimmten Rhythmus realisieren. Dabei wird sie aber als routinierte, das heißt wiederkehrende, und von mehreren Perso- 
nen oder Praxisgemeinschaften (communities of practice) geteilte Art des Umgangs mit Zeit begriffen.

\subsection{Zeitlichkeit von Praxis, Zeitlichkeit von Praktiken, Zeitpraktiken}

Was heißt das für die Analyse der Bearbeitung von Zukunft? Wir möchten das hier in drei Punkten andeuten. Beginnen wir grundsätzlich: mit dem Verhältnis von Zeit und Praxis. Rücken Praktiken und Zukunft in den Fokus des Interesses, dann stellt sich die Frage, auf welcher grundsätzlichen Ebene dieses Verhältnis zu analysieren ist. Hierzu bietet es sich an, die uns interessierenden Zukunftspraktiken von zwei anderen Ebenen zu unterscheiden. Aus praxistheoretischer Perspektive lässt sich die Zeitlichkeit des Sozialen auf drei Ebenen ausmachen: auf der der Zeitlichkeit sozialer Praxis generell, auf jener der Zeitlichkeit der einzelnen sozialen Praktik und schließlich auf jener der „Zeitpraktiken“ im engeren Sinne (vgl. Reckwitz 2016).

Die erste Ebene betrifft die Zeitlichkeit sozialer Praxis insgesamt. Gemeint ist damit eine grundsätzliche Zeitlichkeit von Praxis, welche selbst immer schon zeitlich strukturiert ist. Die Praxis ist eine Sequenz von Ereignissen, die eine Zukunft und Vergangenheit haben, indem sie auf Vorheriges Bezug nehmen und ihnen auch Nachgängiges folgt. Als ,,a temporally unfolding and spatially dispersed nexus of doings and sayings" (Schatzki 1996, S. 89) wird soziale Praxis damit immer als ein Strom von Ereignissen begriffen, die ein Vorher und Nachher haben. In der Wiederaufführung eingeübter körperlicher Akte bildet die soziale Praxis einen Bezug zur Vergangenheit aus. Sie ist in der situativen Aufführung allerdings immer wieder kleinen und auch großen Veränderungen unterworfen, die Möglichkeit bieten für Neues - das heißt auch für Änderungen der jeweiligen Praxis. Garfinkel (1967, S. 9) nennt das ,for another first time“ oder in einem späteren Text heißt es: ,,another next first time" (Garfinkel 2002, S. 182).

Im Gegensatz zu dieser grundsätzlichen Temporalität sozialer Praxis ist eine zweite Ebene zu unterscheiden: die Zeitlichkeit einzelner sozialer Praktiken. So wie jede Praxis grundsätzlich eine zeitliche Dimension hat, so lassen sich einzelne Praktiken durch ihre ganz spezifische Zeitlichkeit unterscheiden. Es geht dann um die spezifische Zeitlichkeit, die die jeweilige Praxis hervorbringt, nicht um ihre grundsätzliche sozialontologische Ereignisstruktur. Hier lassen sich zwei Formen unterscheiden: Zum einen folgen spezifische Praktiken auf gewisse Ereignisse bzw. gehen diesen voran. In einer Sitzung des Vorstandes etwa können die Grundlagen für betriebliche Veränderungen gelegt werden, ebenso aber kann die Vorstandssitzung die Reaktion auf betriebliche Probleme darstellen. Sie sind in diesem Sinne auch immer ein Vorgriff auf Kommendes, zugleich aber auch ein Verweis auf Vergangenes, indem sie auch Ergebnis bereits vollzogener Praktiken sind. Beide temporalen Verweise in die Zukunft und die Vergangenheit - erschöpfen sich jedoch gerade nicht in der unmittelbaren zeitlichen Nähe, sondern sie verweisen noch viel weiter. So kann eine Sitzung von Vorständen erst viele Jahre später als entscheidendes Ereignis begriffen werden (vgl. Jarzabkowski et al. 2007) und gleichzeitig weist die kulturelle Praxis des Sitzungsabhaltens weit in die Geschichte kooperativer Auseinandersetzungen zurück (vgl. Sennett 2012). Zum anderen strukturieren Praktiken Zeit selbst auf eine spezifische Art und Weise. Beispielsweise wird bei der Durchführung von Vor- 
standssitzungen zunächst zwischen Rede- und Schweigezeiten unterschieden, welche wiederum verschiedenen Akteuren zugewiesen werden. Darüber hinaus werden ein genauer Anfang und ein ungefähres Ende festgelegt. Auch sind die Sitzungen in ihrem dramaturgischen Ablauf nicht selten so organisiert, dass einzelne inhaltliche Höhepunkte von anderen weniger wichtigen Aspekten getrennt werden (vgl. Jarzabkowski und Seidl 2008). Vorstandssitzungen verfügen damit über einen spezifischen zeitlichen Rhythmus, der sie strukturiert.

Von diesen beiden Formen zu unterscheiden ist schließlich ein dritter Typus des Zusammenhangs von Zeit und Praxis, der für unsere Frage nach der konkreten Gestalt(ung) von Zukunft eine herausgehobene Rolle spielt. Es handelt sich um diejenigen Praktiken, die dezidiert auf Zeit bezogen sind, die sogenannten ,temporalen Praktiken“ (Kaplan und Orlikowski 2013), d. h. die Zeitpraktiken (Hörning et al. 1997) im engeren Sinne. Ein prominentes Beispiel in den Sozial- und Wirtschaftswissenschaften etwa sind Praktiken des Erinnerns (vgl. Assmann 2013; Feldman und Feldman 2006; Gioia et al. 2002). Dies meint dann die Routinen und Prozeduren, mithilfe derer an vergangene Ereignisse als geschehene Ereignisse angeknüpft wird - auch wenn diese in die Gegenwart hinein verlagert werden, mit dem mal mehr oder minder erklärten Ziel, in die Zukunft zu wirken. Dazu gehören das Ausführen von organisationsinternen Erinnerungsritualen wie etwa das Abhalten von Gedenkveranstaltungen zum Firmenjubiläum sowie das Erstellen von Filmen oder Büchern, in denen an vergangene Ereignisse wie z. B. die Gründung der Organisation erinnert werden soll. Konkordant dazu sind „Zukunftspraktiken“ folglich diejenigen Arten und Weisen, welche dezidiert darauf aus sind, einen expliziten Bezug zum Zeitmodus des Kommenden herzustellen. Es geht also um die Vorausschau oder die Hereinnahme und Bearbeitung zukünftiger Ereignisse oder Gegebenheiten in der Gegenwart.

\subsection{Zukunftspraktiken: Performativität, Situativität, Heterogenität und Relationalität}

Die hier angedeuteten Grundlagen einer Theorie sozialer Praxis implizieren vier analytische Dimensionen, die in praxistheoretischen Untersuchungen zur zeitgenössischen Bearbeitung des Kommenden zum Tragen kommen. Diese gilt es im Folgenden zu beschreiben und ihre Rolle bei der Analyse von Zukunftspraktiken zu verdeutlichen.

Erstens macht eine praxistheoretische Perspektive die Performativität von $\mathrm{Zu}$ kunft deutlich. Indem ein derartiger analytischer Zugriff die konkreten Praktiken in den Blick nimmt, mithilfe derer Zukunft innerhalb von Organisationen hervorgebracht wird, lenkt sie die Aufmerksamkeit auf das (Un-)Doing von Zukunft. Dies bedeutet zugleich, dass eine Analyse der Zukunftsbearbeitung nicht nur danach fragt, wie Zukunft imaginiert und verarbeitet wird, sondern auch, welche Zukunft dabei entsteht. Während beispielsweise der Vollzug prognostisch-probalistischer Verfahren Zukunftsprojektionen aus Vergangenem konstruiert, setzen visionäre Zukunftserzählungen hingegen gerade explizit auf den Bruch mit gegenwärtigen und bekannten Erfahrungen. Beide Verfahren lassen differente Vorstellungen über das Kommende entstehen, denen es sich gegenüber zu verhalten gilt, d. h. die sowohl aktiv als auch 
passiv ertragen, erwünscht, abgewehrt etc. werden müssen. Die Zukunftsimagination wird damit zur ,force for action“ (Beckert 2013, S. 232), d. h., durch Handlungsvollzüge wird das Kommende als performatives Ereignis, als Aus- und Aufführung deutlich.

Damit auf das Engste verknüpft ist eine zweite Dimension - nämlich die der Situativität. Mit einer praxistheoretischen Perspektive auf Zukunft wird das Kommende zunächst als gegenwärtiges Phänomen untersucht. Es geht um die Arten und Weisen, mit denen Zukunft in der Gegenwart hervorgebracht und verarbeitet wird. Dies ist in der Auseinandersetzung mit Zeit keineswegs ein Novum. Bereits in der für die Organisationsforschung bedeutsamen philosophischen Strömung des Pragmatismus (Mead 1932) oder die im deutschsprachigen Raum einflussreiche Theorie funktionaler Differenzierung (Luhmann 1984) verweisen auf derartige „gegenwärtige Zukünfte“ (Luhmann 1976). Zugleich geraten aber auch so „,zukünftige Gegenwarten“ in den Blick, welche als Imaginationen, Erwartungen, Prognosen etc. aber immer an die Gegenwart gebunden sind. Ein derartige Betonung der Situativität wird oft mit dem Vorwurf verbunden, dass Praxistheorien sich auf einen Situationalismus beschränken würden, der keine Makro- oder Gesellschaftsstrukturen in den Blick nimmt (vgl. dazu Seidl und Whittington 2014). Hierzu ist jedoch anzumerken, dass Praxistheorien die konkrete Handlungssituation keineswegs von übersituativen Bezügen entkoppeln, sondern diese anders verorten: eben nicht der Praxis vorgängig, sondern fundamental mit dieser verschränkt (vgl. bereits Giddens 1984). Wenn entsprechend übersituative Bezüge, seien dies Organisationskulturen, Weisungsbefugnisse/Hierarchien, tradierte Leitbilder, oder auch größere Ordnungsprinzipien (wie etwa der Taylorismus) eine Rolle spielen, dann finden diese eben auch in der Praxis ihren Niederschlag.

Drittens ist die Heterogenität der Praxis von entscheidender analytischer Bedeutung. Indem der Ausgangspunkt der Analyse organisationaler Zukünfte auf Praktiken und nicht davon losgelösten, übergeordneten Zukunftskonzepten liegt, gerät auch die empirische Vielfältigkeit der Zukunftspraxis in den Blick. Eine praxistheoretische Perspektive fokussiert damit nicht nur die Analyse von Praktiken, mit denen explizit Zukunft bearbeitet wird, sondern eben auch jener Verfahren, die nicht von vornherein explizit auf Zukunft hin ausgerichtet sind, aber letztlich auch einen deutlichen Einfluss auf die Zukunftsgestaltung haben. Das Raster für die Identifikation derartiger Zukunftspraktiken liefern damit nicht vorgängige Theorieentscheidungen; erst durch eine empirische Praxisanalyse ist zu entscheiden, welche Praktiken Zukunft bearbeiten. Eine solche Perspektive nimmt daher die Möglichkeit der Überraschung als Analysedimension ernst. So lassen sich in Organisationen möglicherweise multiple Umgänge mit Zukunft identifizieren - und damit ebenso verschiedene Zukünfte.

Verknüpft mit der Heterogenität der Zukunftsbearbeitung verweist eine praxistheoretische Perspektive, viertens, auf die Relationalität derartiger Praktiken. Das Verständnis der Praxis als ein „Nexus“ (Schatzki 1996) und somit als das Zusammenspiel unterschiedlicher Aktivitätsströme verweist darauf, dass Praktiken niemals einzelne und nur für sich stehende Entitäten, sondern selber in ein Netz von Praktiken prozessual eingebunden sind (vgl. z. B. Deken et al. 2016; Kremser und Schreyögg 2016). 
Daraus ergeben sich für die Praxistheorie einige Analysekategorien von besonderem Interesse. Zunächst legt die Praxistheorie einen besonders Fokus auf die Materialität des Sozialen und dies in einem doppelten Sinne. Sie interessiert sich für die Artefakte und Körper der Praxis. Als routinierte Wiederaufführungen sind Praktiken maßgeblich auf kompetente Körper angewiesen, die sie hervorbringen. „Wenn ein Mensch eine Praktik erwirbt, dann lernt er, seinen Körper auf bestimmte, regelmäßige und ,gekonnte“ Weise zu bewegen und zu aktivieren oder besser: auf eine bestimmte Art und Weise Körper zu „sein“, da der Körper aus praxeologischer Perspektive kein ausführendes Instrument darstellt, das von einem „,dahinter liegenden“ Zentrum gesteuert würde“ (Reckwitz 2003, S. 290). Ebenso spielen Artefakte eine herausgehobene Rolle, da der Umgang mit ihnen gewisse Praktiken maßgeblich strukturiert und anleitet. Träger von Praktiken sind damit nicht nur menschliche Akteure, sondern auch Objekte, die vorhanden sein müssen, damit eine Praxis vollzogen und reproduziert werden kann. Für Zukunftspraktiken bedeutet das, mithilfe spezifischer körperlicher Vollzüge und unter maßgeblichem Einbezug von Artefakten Zukünfte hervorzubringen. Etwa wird in den wöchentlichen Sitzungen auf Leitungsebene der Körper ganz anders eingesetzt als bei Formen der Zukunftsbearbeitung mittels schamanischer Zeremonien. Auch die Artefakte unterscheiden sich maßgeblich - während in dem einen Fall die ubiquitäre (post-)moderne Büroausstattung zu finden ist, sind es im anderen Objekte jenseits der Arbeitswelt, die als vermeintlich „,natürliche“ Dinge die Praxis anleiten.

Neben dieser Materialitätsdimension werden Praktiken maßgeblich durch einen „praktischen Sinn“ (Bourdieu 1976, 1987) angeleitet, der nur im Vollzug selbst beobachtet werden kann. Der Sinn einer einzelnen Praxis findet sich also in ihrer Aus- und Aufführung, wobei diese maßgeblich mit kulturellen Artefakten und Symbolen verbunden ist. Dieser prozessuale Sinnbegriff drängt die Forschenden erneut zur eingehenden Analyse konkreter Situationen in actu. Zugleich ist damit auf kollektive Wissensordnungen verwiesen, die mit diesem praktischen Sinn fundamental verbunden sind. Eine Praxis verweist über einen geteilten Wissenshorizont auf die praktisch-sinnhaften Regeln, die in ihrem Vollzug ihre ordnungsleistende Kraft zeitigen. Dass ein Unternehmen etwa seine Zukunft nach planerischen Wissensprinzipien strukturiert, zeigt sich dann nicht im Organigramm der Organisation, sondern im know-how-abhängigen Wissen der ausgeführten Praktiken (vgl. z. B. Danner-Schröder und Geiger 2016); also in der Erstellung des Plans, in der Einhaltung und Überwachung der daraus resultierenden Anweisungen zeigt sich das planerische Wissen.

Eine interessante Sonderform von Zukunftspraktiken findet sich in Zeitdiskursen innerhalb von Organisationen. Aus praxistheoretischer Sicht sind Diskurse als diskursive Praktiken ein Spezialfall von Praktiken (vgl. dazu auch Reckwitz 2008). Sie sind als Praktiken der Repräsentation zu verstehen, in denen Zeichensysteme, Argumentationen, Narrationen und Visualitäten hergestellt werden und zirkulieren. Es geht im Fall von Zukunftsdiskursen um die spezifische diskursive Thematisierung, um die Formationsregeln des Repräsentierens von Zukunft. Derartige Zukunftsthematisierungen finden sich in den Leitbildern von Organisationen, den Ansprachen zum Firmenjubiläum, in den Prognostiken der F\&E-Abteilung aber auch in populärwissenschaftlichen Futurologien. Für die praxistheoretische Analyse sind derartige 
Zukunftsdiskurse als Bestandteil von Praxis-/Diskurskomplexen relevant, in denen sie als diskursive und nicht-diskursive Praktiken miteinander verwoben sind. So werden etwa die niedergeschriebenen, wissenschaftlichen Prognosen zur materiellen Grundlage von Diskussionen über die Zukunft einer Organisation - sowohl als apokalyptische Schreckensmeldung für die einen oder hoffnungsfrohe Botschaft für die anderen. Wichtig ist, dass solche Diskurse dabei nicht als bloße Kommentare verstanden werden, sondern eben als ein Bestandteil der spezifischen Praxis der Organisation, als „ein Raum expliziten und thematisierten Wissens“ (Reckwitz 2016, S. 127; vgl. dazu auch Dittrich et al. 2016).

Relational gedacht sind Zukunftspraktiken nun immer als Gemengelage dieser einzelnen Elemente zu betrachten. Eine Praxis verfügt immer über eine materiale Dimension, welche sich aus verschiedenen Artefakten und Körpern zusammensetzt, die im Vollzug die Praxis selbst erst hervorbringen. Dabei ist sie häufig mit mächtigen Diskursen verknüpft, durch einen praktischen Sinn strukturiert und verweist somit auf kollektiv geteilte Wissensordnungen. Aber Praktiken sind nicht nur als punktuelle Gefüge zu beobachten, sondern sind in ihren Relationen immer auch mit anderen Praktiken prozessual verbunden. Die These von der Relationalität der Praxis eröffnet ein Netzwerk an zahlreichen Praktiken, die gemeinsam wiederum Praktiken stabilisieren. So fordern gewisse Praktiken - etwa die Erstellung eines Planes - wiederum andere Praktiken ein. In der öffentlichen Verwaltung ist dies beispielsweise als Planfeststellungsverfahren inzwischen fest institutionalisiert und rechtlich geregelt. Aber auch in weniger reglementierten Bereichen ist eine ritualisierte Ausführung verknüpfter Praktiken zu beobachten. Beispielsweise fordern Firmenjubiläen spezifische wiederkehrende Praktiken ein - etwa das Halten einer Rede, das Beschwören einer Vision, das Abhalten eines Festaktes -, worüber dann wiederum in der Mitarbeiterzeitschrift oder am Frühstückstisch im Pausenraum berichtet wird. Diese Relationen einzelner Praktiken lassen sich auch historisch verfolgen. So lassen sich etwa gewisse ,Zitate“ vergangener Praktiken identifizieren, an die bewusst oder nicht-bewusst angeschlossen wird.

\section{Ausblick: Zur Zukunft der Zukunftsbearbeitung}

Die bisherige Betrachtung bietet eine Reihe von Anhaltspunkten dafür, dass die Praxistheorie einen aufschlussreichen Blick auf die Zukunftsbearbeitung in Organisationen bieten kann. Mit ihrer Fokussierung auf den situativen und mit materialen Objekten, Diskursen und Wissensordnungen relational verschränkten Handlungsvollzug, durch den Zukünfte performativ hervorgerufen werden, eröffnet die praxistheoretische Perspektive die Möglichkeit, die Vielschichtigkeit und Vielseitigkeit möglicher Praktiken der Zukunftsbearbeitung zu analysieren und besser zu verstehen. Welche Praktiken als Zukunftspraktiken gelten, ist dabei zunächst empirisch offen. In den Blick geraten gleichermaßen klassische Steuerungsinstanzen wie beispielsweise Leitungs- und Planungsrunden, aber ebenso alltägliche Aktivitäten wie Fußballspielen oder Betriebsfeiern. Obgleich ein praxistheoretischer Zugang zur Zukunftsbearbeitung eine Vielzahl von Praktiken in den Blick nimmt, die sich weit über bekannte planerische Handlungen hinaus erstreckt, sind die Unterschiede 
von Zukunftspraktiken und Zukunftsformen keineswegs endlos. Die Praxis der Zukunftsbearbeitung ist eben nicht nur ein an spezifische Situationen gebundener Akt, sondern sie (re-)produziert auch geteilte Zukunftspraktiken, die sich sowohl über Situationen als auch über Organisationen hinweg als (wieder-)erkennbare Muster identifizieren lassen.

Dass und in welcher Weise empirische Untersuchungen der vielseitigen und komplexen Praktiken der Zukunftsbearbeitung neue steuerungstheoretische Impulse setzen können, soll hier kurz unter Bezugnahme auf das Burgelman'sche (2002) Strategieprozess-Modell illustriert werden (vgl. dazu auch Burgelman 1983; Burgelman und Grove 2007). Wie oben bereits angeführt, unterscheidet Burgelman bekanntlich zwischen zwei strategischen Handlungstypen: Induced strategic actions basieren auf dem gegenwärtigen strategischen Konzept eines Unternehmens und reflektieren dessen intendierte strategische Entwicklung. Autonomous strategic actions hingegen operieren aus der Perspektive des Gesamtsystems betrachtet losgelöst und damit jenseits des intendierten strategischen Konzepts. Beide Handlungstypen werden von Burgelman in einem wechselseitigen Spannungsverhältnis gedacht, welches sich im Strategieprozess so entfalten sollte, dass keine der beiden strategischen Handlungstypen alleine die strategische Trajektorie eines Unternehmens oder einer Organisation bestimmen sollte (vgl. Burgelman und Grove 2007).

Eine zukunftsinteressierte Betrachtung dieses Strategieprozess-Modells legt nahe, dass die beiden Kategorien strategischer Handlungen eng mit unterschiedlichen $\mathrm{Zu}-$ kunftsimaginationen verknüpft sind. Induced strategic actions sind in das ,strategic planning system of the firm" (Burgelman 1983, S. 65) eingebunden und projizieren daher im Zeitablauf verstärkt das Vergangene in die Zukunft. So nahm Intels CEO, Andy Grove, mit dem zunehmenden Erfolg des Unternehmens im Geschäft mit Mikroprozessoren an: „Intel's future lay[s] in microprocessors“ (Burgelman 2002, S. 331). Autonomous strategic actions hingegen forcieren alternative Zukünfte, die sich nicht mit dem gegenwärtigen strategischen Konzept abbilden lassen. Beispielsweise stellte Grove mit der abnehmenden Bedeutung von strategischen Initiativen jenseits des Geschäfts mit Mikroprozessoren fest: „Intel's future [...] also depend[s] on new business development" (Burgelman 2002, S. 361).

Die damit einhergehende dichotom gedachte Kategorisierung strategischer Handlungen als ,autonom“ oder ,induziert“ (vgl. z. B. Mirabeau und Maguire 2014) stellt letztlich insbesondere empirische Untersuchungen aufgrund der ex ante kaum identifizierbaren und ex post meist als intendiert rationalisierten emergenten Prozesse (vgl. Burgelman 1983; Mintzberg und Waters 1985) vor besondere methodische Herausforderungen. Eine praxistheoretische Perspektive ermöglicht es nun, diese rein dichotome Vorstellung der strategischen Handlungstypen zu unterlaufen und damit insbesondere ihr in der bisherigen Forschung lediglich als Kontrast bestimmtes Verhältnis viel nuancierter und feinkörniger zu bestimmen. Sie tut dies, indem sie die konkreten Konfigurationen und Ausprägungen der Zukunftsbearbeitung im Hinblick auf ihre Performativität, Situativität, Heterogenität und Relationalität bestimmt. Die situative Praxis, in der induzierte und autonome strategische Handlungen vollzogen und damit Zukünfte performativ hervorgerufen werden, fand bisher nur in geringem Maße Beachtung. Die Heterogenität von Zukunftspraktiken lässt darauf schließen, dass derartige empirische Untersuchungen auf ein Bündel von Handlungen stoßen 
werden, die zwischen den Extremtypen von induced und autonoumos einzuordnen sind und deren Exploration gerade in Bezug auf die Frage, welche Zukünfte und damit verbunden, welche Umwelten ein System performativ aktualisiert, wesentlich ist. In diesem Sinne ermöglicht die Perspektive auf die situative Praxis der Zukunftsbearbeitung einen Zugang zum strategischen und organisationalen Kontext eines Unternehmens, der nicht auf die ideal gedachte Trennung, sondern auf das real zu bestimmende Verhältnis der beiden strategischen Handlungstypen abstellt. Insofern verspricht der empirische Blick auf die Relationalität der Zukunftspraxis Erkenntnisse zum Verhältnis von induzierten und autonomen strategischen Handlungen zueinander und legt nahe, das Verhältnis nicht vor dem Hintergrund eines Kontrastes, sondern im Hinblick auf die Form ihrer praktischen Vernetzung hin zu begreifen.

Eine praxistheoretische Untersuchung der Zukunftsbearbeitung in Organisationen ermöglicht damit auch Einblicke in die Praxis der Nicht-Steuerung und geht damit über den Gebrauch von Metaphern (vgl. z. B. McCabe 2016) hinaus. Denkbar ist beispielsweise, dass autonomous strategic actions zwar alternative Zukünfte forcieren, aber diese wiederum nicht zu stark von der durch induced strategic actions hervorgebrachten Zukunft abweichen dürfen, um anschlussfähig zu bleiben und sich so in den strategischen Diskurs einschreiben zu können. Aus dieser Perspektive würde nicht nur die Abwesenheit und Unterdrückung von autonomous strategic actions (vgl. z. B. Burgelman 2002), sondern auch die Radikalität der durch sie hervorgebrachten Zukunftsimaginationen zur strategischen Persistenz von Unternehmen beitragen. So könnte ein Abriss alternativer Zukünfte von der dominant hervorgebrachten Zukunft zur Wahrnehmung von Paradoxien (vgl. Ortmann 2003; Smith und Lewis 2011) und eine zu enge Anbindung zu pfadabhängigen Tendenzen (vgl. Sydow et al. 2009) führen. Diese empirische Offenheit eines praxistheoretischen Zugangs kann daher auch dazu beitragen, etwas Drittes (vgl. Ortmann 2015), d. h. völlig neue Formen von Management zu explorieren, die von planungstheoretischen Vorstellungen entkoppelt sind und möglicherweise dazu verhelfen, Unternehmenssteuerung neu zu denken.

\section{Schlussbetrachtung}

Der vorliegende Beitrag setzte sich zum Ziel, eine praxistheoretische Perspektive auf die Bearbeitung von Zukunft in Organisationen einzuführen und deren Attraktivität zu verdeutlichen. Der Rückblick auf die Entwicklung gesellschaftlicher sowie organisationaler Zeitverständnisse hat gezeigt, dass die in der Spätmoderne eingetretene Kontingenzerfahrung linear-rationale, planungsorientierte Formen der $\mathrm{Zu}$ kunftsbearbeitung zunehmend problematisiert. Dass derartige Verfahren in der Managementforschung weiterhin eine dominante Stellung einnehmen, verdeutlicht die Relevanz eines Forschungsprogramms, das die vielfältigen Figurationen untersucht, durch die zeitgenössische Organisationen Zukünfte vergegenwärtigen und verarbeiten und die sich über planerische Handlungen hinaus erstrecken. Die eingeführten Begrifflichkeiten einer praxistheoretischen Perspektive auf die Zukunftsbearbeitung erweist sich für dieses Forschungsprogramm als besonders nützlich: Erstens rich- 
tet ein praxistheoretischer Ansatz den Blick auf den performativen und situativen Handlungsvollzug, durch den soziale Akteure Zukunftsvorstellungen kreieren und verarbeiten. Er fokussiert somit die gegenwärtige Bearbeitung des Kommens und verhilft zu tiefergehenden Erkenntnissen über die konkreten Aktivitäten, durch die Zukunft imaginiert und verarbeitet wird. Zweitens zeigt sich eine praxistheoretische Perspektive empirisch offen für die heterogenen und mitunter vielfältigen Formen der Zukunftsbearbeitung. Sie bietet damit die Möglichkeit, Planung als einen Hort vermeintlich sicherer Zukunftsbearbeitung zu verlassen und andere - womöglich völlig neue - Praktiken der Zukunftsbearbeitung zu explorieren. Drittens hebt eine praxistheoretische Perspektive die Relationalität von Zukunftspraktiken hervor, d. h. das Zusammenspiel von Zukunftspraktiken mit anderen sozialen Praktiken sowie deren Gefüge aus materialen Trägern, impliziten Wissensordnungen und Diskursen im Handlungsvollzug. Trotz ihres überschaubaren Begriffsvokabulars verdeutlicht sie damit die Komplexität der Zukunftsbearbeitung und stellt einen fundierten Zugang bereit, diese analytisch zu entfalten. Wie im Ausblick schematisch gezeigt, kann eine praxistheoretische Untersuchung der Zukunftsbearbeitung zu mehr als „nur“ der Exploration eines weiteren Bündels an Praktiken verhelfen. Aufgrund der immanenten Rückbindung von Steuerungshandlungen an das Kommende können tiefergehende Erkenntnisse zu konkreten Zukunftspraktiken und deren Zusammenspiel neue steuerungstheoretische Impulse setzen, die Unternehmenssteuerung auch jenseits planerischer Handlungen begreifen und mitunter neu denken.

Mit diesen Überlegungen bringt dieser Aufsatz mehrere Beiträge zur Managementforschung hervor. Erstens werden die Herausforderungen der in der Managementforschung weiterhin dominierenden Idee einer planungszentrierten Zukunftsbearbeitung aufgezeigt. Damit werden frühere allgemeine Verweise auf die Fallibilität von Plänen (vgl. z. B. Barry und Elmes 1997; March 1995; Mintzberg 1994) mit einer Zukunftsperspektive komplementiert und vertieft, die die spätmoderne Kontingenzerfahrung in den Mittelpunkt stellt. Der Aufsatz schafft damit Gehör für neuere Ansätze der Unternehmenssteuerung, die auch die Nichtsteuerbarkeit von Unternehmen konzeptionalisieren (vgl. z. B. Burgelman 2002; Kasper et al. 1999; Schreyögg 1991; Willke 2001) und eröffnet ein Programm zur Untersuchung jener Formen der Zukunftsbearbeitung, die sich nicht in planerischen Handlungen erschöpfen.

Zweitens wird eine praxistheoretische Perspektive auf die Bearbeitung von $\mathrm{Zu}$ kunft vorgeschlagen. Der vorliegende Beitrag geht dabei über eine Einführung des praxistheoretischen Vokabulars hinaus, indem wiederholte Missverständnisse zur Praxistheorie, z. B. zur Rolle von Individuen, Materialität, Diskursen und höheren Ordnungen in der Praxis, aufgegriffen und geklärt werden. Darüber hinaus wird ein konkreter Bezug der Praxistheorie zur Untersuchung von Zukunftspraktiken hergestellt. Damit eröffnet der Beitrag einen praxistheoretischen Zugang zur Analyse und Untersuchung von Zukunftspraktiken hinsichtlich ihrer Performativität, Situationalität, Heterogenität und Relationalität.

Drittens zeigt der Aufsatz auf, was eine praxistheoretische Analyse von Zukunftspraktiken zu leisten vermag. Da der Umgang mit einer unwissbaren Zukunft gegenwärtiges Handeln maßgeblich bestimmt und antreibt, ist eine empirische Analyse der Zukunftspraxis per se ein wichtiger Beitrag zum besseren Verständnis des Verhaltens in und von Organisationen. Aufgrund der Zukunftsgewandtheit von Steuerungshand- 
lungen in Organisationen können derartige Forschungsergebnisse darüber hinaus zu einer Weiterentwicklung steuerungstheoretischer Überlegungen führen, die sich von der Zentrierung planerischer Handlungen lösen und Unternehmenssteuerung neu denken.

\section{Literatur}

Abdallah C, Langley A (2014) The double edge of ambiguity in strategic planning. J Manag Stud 51(2):235-264

Adam B (1990) Time and social theory. Polity, Cambridge

Alvarez SA, Barney JB (2007) Discovery and creation: alternative theories of entrepreneurial action. Strateg Entrep J 1(1-2):11-26

Ancona DG, Okhuysen GA, Perlow LA (2001) Taking time to integrate temporal research. Acad Manage Rev 26(4):512-529

Ansoff HI (1965) Corporate strategy: an analytic approach to business policy for growth and expansion. McGraw-Hill, New York

Arend RJ (2015) Mobius' edge: infinite regress in the resource-based and dynamic capabilities views. Strateg Organ 13(1):75-85

Assmann A (2013) Ist die Zeit aus den Fugen. Aufstieg und Fall des Zeitregimes der Moderne. Hanser, München

Augier M, Teece DJ (2008) Strategy as evolution with design: the foundations of dynamic capabilities and the role of managers in the economic system. Organ Stud 29(8/9):1187-1208

Bakken T, Holt R, Zundel M (2013) Time and play in management practice: an investigation through the philosophies of McTaggart and Heidegger. Scand J Manag 29(1):13-22

Barry D, Elmes M (1997) Strategy retold: toward a narrative view of strategic discourse. Acad Manag Rev 22(2):429-452

Beck U (1986) Risikogesellschaft. Auf dem Weg in eine andere Moderne. Suhrkamp, Frankfurt a. M.

Beckert J (1996) What is sociological about economic sociology? Uncertainty and the embeddedness of economic action. Theory Soc 25:803-840

Beckert J (2013) Imagined futures: fictional expectations in the economy. Theory Soc 42(3):219-240

Beckert J (2016) Imagined futures: expectations and capitalist dynamics. Harvard University Press, Cambridge

Bluedorn AC (2002) The human organization of time: temporal realities and experience. Stanford University Press, Stanford

Böhle F, Pfeiffer S, Sevsay-Tegethoff, N (2004) Die Bewältigung des Unplanbaren. Springer VS, Wiesbaden

Boje DM (1995) Stories of the storytelling organization: a postmodern analysis of Disney as ,Tamaraland“. Acad Manage J 38(4):997-1035

Bourdieu P (1976) Entwurf einer Theorie der Praxis auf der ethnologischen Grundlage der kabylischen Gesellschaft. Suhrkamp, Frankfurt a. M.

Bourdieu P (1987) Sozialer Sinn. Kritik der theoretischen Vernunft. Suhrkamp, Frankfurt a. M.

Brinckmann J, Sung MK (2015) Why we plan: the impact of nascent entrepreneurs' cognitive characteristics and human capital on business planning. Strateg Entrep J 9(2):153-166

Bröckling U (2008) Vorbeugen ist besser ... Zur Soziologie der Prävention. Behemoth 1:38-48

Brown SL, Eisenhardt KM (1997) The art of continuous change: linking complexity theory and time-paced evolution in relentlessly shifting organizations. Adm Sci Q 42(1):1-34

Brunsson N (1989) The organization of hypocrisy: talk, decisions, and actions in organizations. Wiley, New York

Burgelman RA (1983) A model of the interaction of strategic behavior, corporate context, and the concept of strategy. Acad Manage Rev 8(1):61-70

Burgelman RA (2002) Strategy as vector and the inertia of co-evolutionary lock-in. Adm Sci Q 47(2):325-257

Burgelman RA, Grove A (2007) Let chaos reign, then reign in chaos - repeatedly: Managing strategic dynamics for corporate longevity. Strateg Manage J 28(10):965-979

Chandler AD Jr. (1962) Strategy and structure: chapters in the history of the American industrial enterprise. MIT Press, Cambridge

Chia R (1995) From modern to postmodern organizational analysis. Organ Stud 16(4):579-604 
Chia R, Holt R (2009) Strategy without design: the silent efficacy of indirect action. Cambridge University Press, Cambridge

Clarysse B, Wright M, Bruneel J, Mahajan A (2014) Creating value in ecosystems: crossing the chasm between knowledge and business ecosystems. Res Policy 43(7):1164-1176

Clegg SR, Kornberger M, Rhodes C (2005) Learning/becoming/organizing. Organization 12(2):147-167

Costas J, Grey C (2014) The temporality of power and the power of temporality: Imaginary future selves in professional service firms. Organ Stud 35(6):909-937

Curnot F, Giroux H, Langley A (2012) The strategic plan as a genre. Discourse Commun 6(1):21-54

Danner-Schröder A, Geiger D (2016) Unravelling the motor of patterning work: toward an understanding of the microlevel dynamics of standardization and flexibility. Organ Sci 27(3):633-658. doi:10.1287/ orsc.2016.1055

Deken F, Carlile PR, Berends H, Lauche C (2016) Generating novelty through interdependent routines: a process model of routine work. Organ Sci 27(3):659-677. doi:10.1287/orsc.2016.1051

Dittrich K, Guérard S, Seidl D (2016) Talking about routines: the role of reflective talk in routine change. Organ Sci 27(3):678-697. doi:10.1287/orsc.2015.1024

Feldman MS, Orlikowski WJ (2011) Theorizing practice and practicing theory. Organ Sci 22(5):1240-1253

Feldman RM, Feldman SP (2006) What links the chain: an essay on organizational remembering as practice. Organization 13(6):861-887

Garfinkel H (1967) Studies in ethnomethodology. Prentice-Hall, Englewood Cliffs

Garfinkel H (2002) Ethnomethodology's program. Working out Durkheim's aphorism. Rowman and Littlefield, Lanham

Giddens A (1984) Die Konstitution der Gesellschaft. Grundzüge einer Theorie der Strukturierung. Campus, Frankfurt a. M.

Gioia DA, Corley KG, Fabbri T (2002) Revising the past (while thinking in the future perfect tense). J Organ Change Manag 15(6):622-634

Guericke S, Koberstein A, Schwartz F, Voß S (2012) A stochastic model for the implementation of postponement strategies in global distribution networks. Decis Support Syst 53(2):294-305

Hardy C, Maguire S (2016) Organizing risk: discourse, power, and ,riskification“. Acad Manage Rev 41(1):80-108

Hawk A, Yeung B (2015) The right speed and its value. Strateg Manage J 36(2):159-176

Hirschauer S (2004) Praktiken und ihre Körper. Über materielle Partizipanden des Tuns. In: Hörning KH, Reuter J (Hrsg) Doing Culture. Neue Positionen zum Verhältnis von Kultur und sozialer Praxis. transcript, Bielefeld, S 73-91

Hodgkinson GP, Healey MP (2011) Psychological foundations of dynamic capabilities: reflexion and reflection in strategic management. Strateg Manage J 32(13):1500-1516

Hodgkinson GP, Wright G (2002) Confronting strategic inertia in a top management team: learning from failure. Organ Stud 23(6):949-977

Hölscher L (1999) Die Entdeckung der Zukunft. Fischer Taschenbuch, Frankfurt a. M.

Hörning KH, Ahrens D, Gerhard A (1997) Zeitpraktiken. Experimentierfelder der Spätmoderne. Suhrkamp, Frankfurt a. M.

Howard-Grenville JA (2005) The persistence of flexible organizational routines: the role of agency and organizational context. Organ Sci 16(6):618-636

Hydle KM (2015) Temporal and spatial dimensions of strategizing. Organ Stud 36(5):643-663

Jarzabkowski P, Balogun J (2009) The practice and process of delivering integration through strategic planning. J Manage Stud 46(8):1255-1288

Jarzabkowski P, Kaplan S (2015) Strategy tools-in-use: a framework for understanding ,technologies of rationality“ in practice. Strateg Manage J 36(4):537-558

Jarzabkowski P, Seidl D (2008) The role of meetings in the social practice of strategy. Organ Stud 29(11):1391-1426

Jarzabkowski P, Balogun J, Seidl D (2007) Strategizing: the challenges of a practice perspective. Hum Relat 60(1):5-27

Joutsenvirta M, Vaara E (2015) Legitimacy struggles and political corporate social responsibility in international settings: a comparative discursive analysis of a contested investment in Latin America. Organ Stud 36(6):741-777

Kaplan S, Orlikowski WJ (2013) Temporal work in strategy making. Organ Sci 24(4):965-995

Kasper H, Mayrhofer W, Meyer M (1999) Management aus systemtheoretischer Perspektive: Eine Standortbestimmung. In: v. Eckardstein D, Kasper H, Mayrhofer W (Hrsg) Management. Schäffer-Poeschel, Stuttgart, S 161-209 
Koch J (2003) Organisation und Differenz. Kritik des organisationstheoretischen Diskurses der Postmoderne. Westdeutscher Verlag, Wiesbaden

Koch J (2011) Inscribed strategies: exploring the organizational nature of strategic lock-in. Organ Stud 32(3):337-363

Koselleck R (1989) Vergangene Zukunft der frühen Neuzeit. In: Koselleck R (Hrsg) Vergangene Zukunft. Zur Semantik geschichtlicher Zeiten. Suhrkamp, Frankfurt a. M., S 17-37

Krämer H (2014) Die Praxis der Kreativität. Eine Ethnografie kreativer Arbeit. transcript, Bielefeld

Kremser W, Schreyögg G (2016) The dynamics of interrelated routines: introducing the cluster level. Organ Sci 27(3):698-721. doi:10.1287/orsc.2015.1042

Luhmann N (1973) Vertrauen: Ein Mechanismus der Reduktion sozialer Komplexität, 2. Aufl. UTB, Stuttgart

Luhmann N (1976) The future cannot begin: temporal structures in modern society. Soc Res (New York) 43(1):130-153

Luhmann N (1984) Soziale Systeme. Grundriß einer allgemeinen Theorie. Suhrkamp, Frankfurt a. M.

March JG (1995) The future, disposable organizations and the rigidities of imagination. Organization 2(3/4):427-440

McCabe D (2016) „Curiouser and curiouser!“: Organizations as Wonderland - a metaphorical alternative to the rational model. Hum Relat 69(4):945-973

Mead GH (1932) The philosophy of the present. Open Court Company, London

Meyer JW, Rowan B (1977) Instutionalized organizations: formal structure as myth and ceremony. Am J Sociol 83(2):340-363

Minois G (1998) Geschichte der Zukunft. Orakel, Prophezeiungen, Utopien, Prognosen. Artemis \& Winkler, Düsseldorf

Mintzberg H (1994) The rise and fall of strategic planning: reconceiving roles for planning, plans, planners. Free Press, New York

Mintzberg H, Waters JA (1985) Of strategies deliberate and emergent. Strateg Manage J 6(3):257-272

Mirabeau L, Maguire S (2014) From autonomous strategic behavior to emergent strategy. Strateg Manage J 35(8):1202-1229

Nadarajah S, Margot F, Secomandi N (2015) Relaxations of approximate linear programs for the real option management of commodity storage. Manage Sci 61(12):3054-3076

Nadkarni S, Chen T, Chen J (2016) The clock is ticking! Executive temporal depth, industry velocity, and competitive aggressiveness. Strateg Manag J 37(6):1132-1153. doi:10.1002/smj.2376

Nag R, Hambrick DC, Chen M-J (2007) What is strategic management, really? Inductive derivation of a consensus definition of the field. Strateg Manage J 28(9):935-955

Nicolini D (2013) Practice theory, work \& organization. An introduction. Oxford University Press, Oxford

Noss C (2013) Strategisches Management und Zeit: Auf dem Weg zu einem integrativen Konzept zeitinduzierter Wettbewerbsvorteile. Managementforschung 23:83-126

Nowotny H (1989) Eigenzeit. Entstehung und Strukturierung eines Zeitgefühls. Suhrkamp, Frankfurt a. M.

Ordóñez LD, Schweitzer MD, Galinsky AD, Bazerman MH (2009) Goals gone wild: the systematic side effects of overprescribing goal setting. Acad Manag Perspect 23(1):6-16

Orlikowski WJ, Yates J (2002) It's about time: temporal structuring in organizations. Organ Sci 13(6):684-700

Ortmann G (2003) Regel und Ausnahme: Paradoxien sozialer Ordnung. Suhrkamp, Frankfurt a. M.

Ortmann G (2013) Noch nicht/nicht mehr: Zur Temporalform von Paradoxien des Organisierens. Managementforschung 23:1-48

Ortmann G (2015) Tertium datur: Figuren des Dritten in der Organisationstheorie (und -praxis). Managementforschung 25:1-40

Pacheco-de-Almeida G, Hawk A, Yeung B (2015) The right speed and its value. Stategic Manage J 36(2):159-176

Reckwitz A (2002) Toward a theory of social practices: a development in culturalist theorizing. Eur J Soc Theory 5(2):243-263

Reckwitz A (2003) Grundelemente einer Theorie sozialer Praktiken. Eine sozialtheoretische Perspektive. Z Soziol 32(4):282-301

Reckwitz A (2008) Praktiken und Diskurse. Eine sozialtheoretische und methodologische Relation. In: Kalthoff H, Hirschauer S, Lindemann G (Hrsg) Theoretische Empirie. Die Relevanz qualitativer Forschung. Suhrkamp, Frankfurt a. M., S 188-209 
Reckwitz A (2016) Zukunftspraktiken. Die Zeitlichkeit des Sozialen und die Krise der modernen Rationalisierung der Zukunft. In: Reckwitz A (Hrsg) Kreativität und soziale Praxis. Studien zur Sozial- und Gesellschaftstheorie. transcript, Bielefeld, S 115-135

Rosa H (2005) Beschleunigung. Die Veränderung der Temporalstrukturen in der Moderne. Suhrkamp, Frankfurt a. M.

Sarasvathy SD (2001) Causation and effectuation: toward a theoretical shift from economic inevitability to entrepreneurial contingency. Acad Manage Rev 26(2):243-263

Schatzki TR (1996) Social practices. A Wittgensteinian approach to human activity and the social. Cambridge University Press, Cambridge

Schatzki TR (2012) A primer on practices: theory and research. In: Higgs J, Barnett R, Billett S, Hutchings M, Trede F (Hrsg) Practice-based education: perspectives and strategies. Sense Publishers, Rotterdam, S 13-26

Schreyögg G (1991) Der Managementprozeß - neu gesehen. Managementforschung 1:17-42

Schreyögg G, Steinmann H (1987) Strategic control: a new perspective. Acad Manage Rev 12(1):91-103

Schultz M, Hernes T (2013) A temporal perspective on organizational identity. Organ Sci 24(1):1-21

Seidl D, Whittington R (2014) Enlarging the strategy-as-practice research agenda: towards taller and flatter ontologies. Organ Stud 35(10):1407-1421

Sennett R (2012) Zusammenarbeit. Was unsere Gesellschaft zusammenhält. Hanser, Berlin

Smith WK, Lewis MW (2011) Toward a theory of paradox: a dynamic equilibrium model of organizing. Acad Manage Rev 36(2):381-403

Song M, Zhao YL, Arend RJ, Im S (2016) Strategic planning as a complex and enabling managerial tool. Strateg Manage J. doi:10.1002/smj.2420

Strohschneider S, van der Weth, R (2002) Ja, mach nur Pläne. Pannen und Fehlschläge - Ursachen, Beispiele, Lösungen. Hogrefe, Bern

Sydow J, Schreyögg G, Koch J (2009) Organizational path dependence: opening the black box. Acad Manage Rev 34(4):689-709

Teece DJ (2007) Explicating dynamic capabilities: the nature and microfoundations of (sustainable) enterprise performance. Strateg Manage J 28(13):1319-1350

Urry J (2000) Sociology beyond societies: mobilities for the Twenty-First Century. Routledge, Oxon

Vaara E, Tienari J (2008) A discursive perspective on legitimation strategies in multinational corporations. Acad Manage Rev 33(4):985-993

Vaara E, Whittington R (2012) Strategy-as-practice: taking social practices seriously. Acad Manag Ann $6(1): 285-336$

Vaara E, Kleymann B, Seristö H (2004) Strategies as discursive constructions: the case of air alliances. J Manage Stud 41(1):1-35

Vaara E, Sorsa V, Pälli P (2010) On the force potential of strategy texts: a critical discourse analysis of a strategic plan and its power effects in a city organization. Organization 17(6):685-702

Wagner P (1994) A sociology of modernity: liberty and discipline. Routledge, London

Weber M (1922) Wirtschaft und Gesellschaft. Mohr Siebeck, Tübingen

Weick KE, Sutcliffe KM (2007) Managing the unexpected: resilience in an age of uncertainty, 2. Aufl. Wiley, San Francisco

Wenzel M (2015) Path dependence and the stabilization of strategic premises: How the funeral industry buries itself. Bus Res 8(2):265-299

Wenzel M, Senf NN, Koch J (2016) Exploring complex phenomena with qualitative research methods: an examination of strategic innovation trajectories in haute cuisine. In: Berger ESC, Kuckertz A (Hrsg) Complexity in entrepreneurship, innovation and technology research: applications of emergent and neglected methods. Springer, Wiesbaden, S 163-182

Willke H (2001) Systemtheorie III: Steuerungstheorie, 3. Aufl. UTB, Stuttgart 